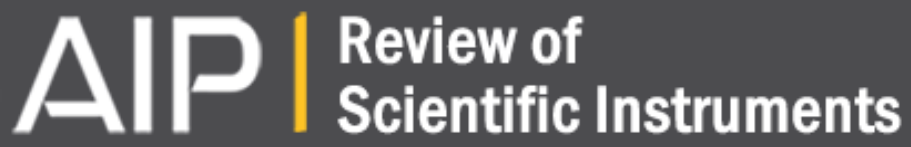

\section{Scanning probe microscope simulator for the assessment of noise in scanning probe microscopy controllers}

T. Wutscher, J. Niebauer, and F. J. Giessibl

Citation: Review of Scientific Instruments 84, 073704 (2013); doi: 10.1063/1.4812636

View online: http://dx.doi.org/10.1063/1.4812636

View Table of Contents: http://scitation.aip.org/content/aip/journal/rsi/84/7?ver=pdfcov

Published by the AIP Publishing

\section{Articles you may be interested in}

Circular mode: A new scanning probe microscopy method for investigating surface properties at constant and continuous scanning velocities

Rev. Sci. Instrum. 82, 113703 (2011); 10.1063/1.3658049

Assessment of the performance of scanning capacitance microscopy for $\mathrm{n}$-type gallium nitride

J. Vac. Sci. Technol. B 26, 611 (2008); 10.1116/1.2890705

Calibrating a tuning fork for use as a scanning probe microscope force sensor

Rev. Sci. Instrum. 78, 063704 (2007); 10.1063/1.2743166

Impacts of probe-tip tilt on scanning probe microscopy

J. Vac. Sci. Technol. B 22, 3394 (2004); 10.1116/1.1813458

Carbon nanotubes as a tip calibration standard for electrostatic scanning probe microscopies

Appl. Phys. Lett. 81, 754 (2002); 10.1063/1.1496129

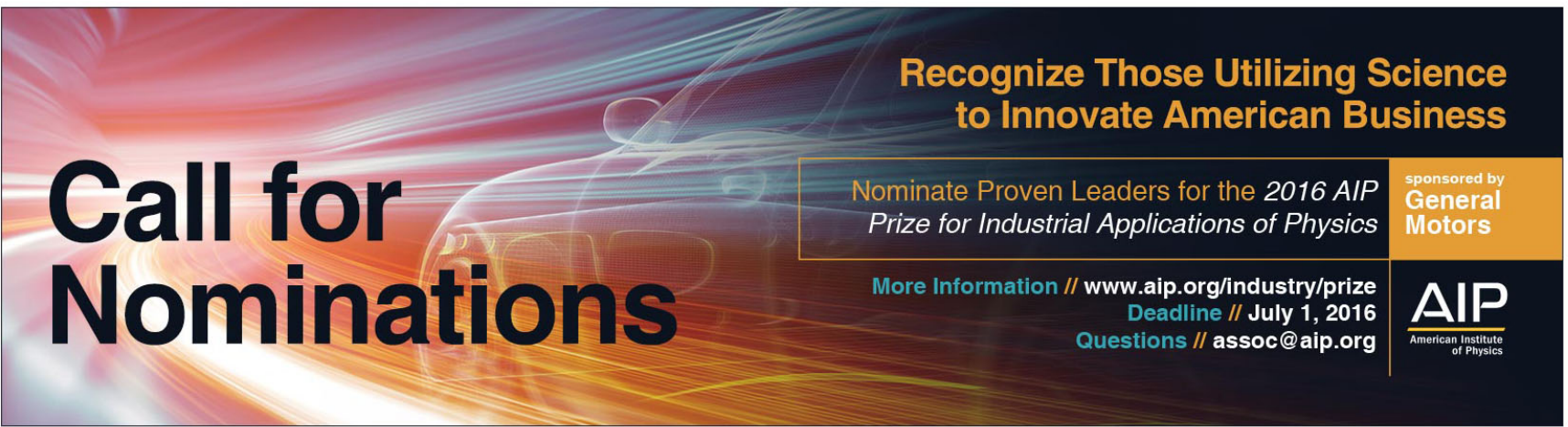




\title{
Scanning probe microscope simulator for the assessment of noise in scanning probe microscopy controllers
}

\author{
T. Wutscher, J. Niebauer, and F. J. Giessib|a) \\ Institute of Experimental and Applied Physics, University of Regensburg, 93040 Regensburg, Germany
}

(Received 9 May 2013; accepted 17 June 2013; published online 9 July 2013)

\begin{abstract}
We present an electronic circuit that allows to calibrate and troubleshoot scanning probe microscopy (SPM) controllers with respect to their noise performance. The control signal in an SPM is typically highly nonlinear-the tunneling current in scanning tunneling microscopy (STM) varies exponentially with distance. The exponential current-versus-voltage characteristics of diodes allow to model the current dependence in STM. Additional inputs allow to simulate the effects of external perturbations and the reactions of the control electronics. We characterized the noise performance of the feedback controller using the apparent topography roughness of recorded images. For a comparison of different STM controllers, an optimal gain parameter was determined by exploring settling times through a rectangular perturbation signal. We used the circuit to directly compare the performance of two types of SPM controllers used in our laboratory. (c) 2013 AIP Publishing LLC. [http://dx.doi.org/10.1063/1.4812636]
\end{abstract}

\section{INTRODUCTION}

When scanning tunneling microscopy (STM) was invented in 1983 by Binnig and Rohrer, the strong variation of tunneling current with distance was soon identified as the foundation of the STMs spectacular spatial resolution. ${ }^{1,2}$ The tunneling of electrons is used to probe the surface of a conductive sample by a conductive tip. In the following years, instrumentation made rapid progress ${ }^{3}$ and feedback circuits that linearize the dramatic distance dependence of STM currents were implemented. ${ }^{4}$ In atomic force microscopy, the short range force that leads to true atomic resolution is also strongly dependent on distance. ${ }^{5-7}$ To reach high resolution, scanning probe microscopes need to be stable mechanically and SPM controllers with low electronic noise are needed.

The SPM controller adjusts the tip-sample distance as a function of the desired control variable-a tunneling current in STM and a force in AFM. The STM controller provides an output voltage $V_{z}$, which changes the distance $z$ between tip and sample. The tunnel current signal increases exponentially with decreasing distance $z$. Keeping the tunnel current signal constant, a closed feedback loop configuration is necessary as schematically shown in Fig. 1. After amplification and a conversion of the tunnel current signal into a voltage by a current-to-voltage $(I / V)$ converter, the signal enters the STM controller. The controller processes the incoming voltage, representing the actual $I_{(x, y, z)}$ signal, and compares this to an adjusted reference value $I_{s e t} . I_{s e t}$ is defined by the user of the STM controller. The error signal $I_{\text {set }}-I_{(x, y, z)}$ regulates the outgoing $z$ voltage, which is applied to a tube scanner.

The magnitude of the error signal depends on the scanning speed and the setpoints of the manually ad-

a)Electronic mail: franz.giessibl@ur.de justed gain parameters of the STM controller. The gain parameters are not standardized, which complicates a comparison between different STM controller systems-some controllers use arbitrary numbers, others gain factors for proportional gain and time or frequency units for integral controllers.

The accuracy of minimizing the error signal is limited by noise. Noise in the STM signal has different causes and can be subdivided in electronic noise and noise associated with the interaction between tip and sample. Since scanning a tip over a surface is not possible without a STM controller, it is difficult to deconvolute the tip-sample noise and the noise associated with the STM controller. If the sample surface and the tip are well prepared, an ideal surface contour is expected on the atomic level. If the topography image of the surface looks noisy, it seems at a first glance to be caused by the tip-sample system. The tip-sample noise is caused by unpredictable events, such as unstable tips, tip changes by collisions with the sample as well as fast contamination of highly reactive tips or samples. The prevention of tip-sample noise caused by e.g. unstable tips yielded in manifold tip preparation methods and for more detailed information we refer the reader to Refs. 8-11. Despite of all these efforts, it can never be excluded that the tip-sample system changes at the atomic level. The irregularities of tip and sample modify an ideal surface contour along atomic corrugations and again complicate the detection of noise in a STM system.

With an ideal tip-sample system, any remaining noise is attributed to noise generated by the SPM controller. This article introduces an electronic device, called scanning probe microscope simulator (SPMS), which models the exponential behaviour of the tunnel current between tip and sample. Simulating the tip-sample junction by a diode eliminates the need to prepare a well characterized and stable tip-sample junction. This allows to deconvolute the tip-sample noise from the noise associated with the STM controller. The SPMS replaces the tip-sample system, enabling for a reproducible control of 


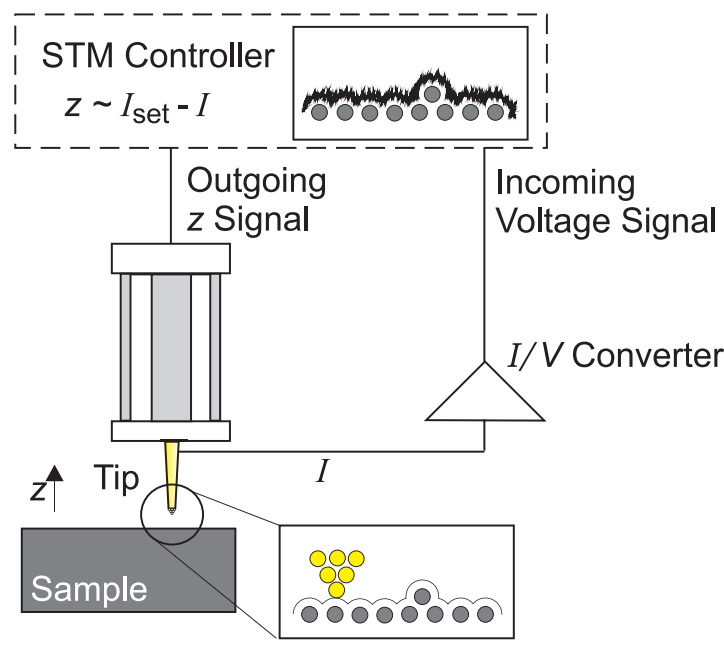

FIG. 1. Schematic view of a STM system. The STM controller adjusts the $z$ position of the tip such that, ideally, the tunneling current matches the setpoint for all $x, y$-positions and generates the $x, y$ positions. ${ }^{4}$ The bottom inset shows the ideal tip trajectory, the top inset displays the actual trajectory that suffers from overlaid noise.

the tunnel current in a closed feedback loop configuration. It also serves as a standardized noise source.

\section{SETUP}

The circuitry of the SPMS and its connection to the STM controller is shown in Fig. 2. The circuit consists of four sections: the input resistors, limitation diodes, an impedance converter, and the tunneling current diode followed by an $I / V$ converter. The input resistors $R_{1}=100 \mathrm{k} \Omega, R_{2}=1 \mathrm{M} \Omega$, and $\mathrm{R}_{3}=10 \mathrm{M} \Omega$ attenuate the incoming signal. They can also be used in parallel to combine input signals. The remaining volt-

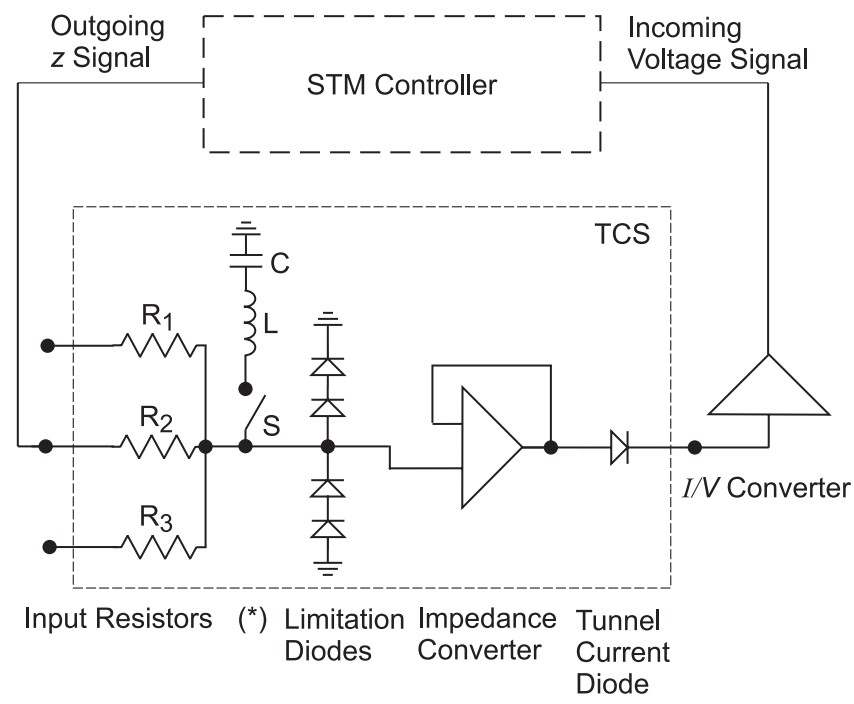

(* optionally an LC series can be added)

FIG. 2. Schematic of the scanning probe microscope simulator (SPMS). The SPMS consists of (from left to right) input resistors, limitation diodes, an impedance converter, and the tunneling current diode. The following currentto-voltage converter can be part of the SPMS or separately attached to the feedback system. Optionally, the dynamic response of the mechanical loop of the microscope can be added by closing switch $\mathrm{S}$, where an LC series is connected to ground. Choosing $L=25 \mathrm{mH}$ and $C=1 \mu \mathrm{F}$ leads to a scanner resonance of $1 \mathrm{kHz}$. age is restricted to $1.3 \mathrm{~V}$ with the limitation diodes $1 \mathrm{~N} 4148 .{ }^{12}$ The impedance converter, based on the AD711 opamp, stabilizes the input signal. ${ }^{13}$ The high conductance, low leakage diode FDLL300 ${ }^{14}$ converts the voltage into a current. The output current of the diode is exponentially dependent on the input voltage, similar to the tunnel current signal between tip and sample. It is important to note that the capacity of the tip-sample junction and the leads that connect it to the preamplifier have a strong influence on noise and bandwidth of the current measurement, where a low capacity is desirable and values of $10 \mathrm{pF}$ or so are considered excellent. The diode type FDLL has a capacity of $6.0 \mathrm{pF}$, thus it does not alter the dynamic response of the simulation circuit significantly. If a higher capacity of the lead connecting the STM to the amplifier is to be modelled, an additional capacity can be connected to ground at the diodes output. The SPMS is designed for positive input voltages, as a positive increase in $V$ corresponds to an exponential increase in $I$.

The $I / V$ converter amplifies the current by a factor of $10^{8} \mathrm{~V} / \mathrm{A}$ to an output voltage. For a truthful simulation of an actual scanning tunneling microscope, it is advisable to use the same $I / V$ converter as in the microscope. The output voltage can be directly connected to the tunnel current input of the STM controller. The outgoing $z$ signal of the STM controller is the input signal of the SPMS.

\section{THE ASSESSMENT OF NOISE BY TOPOGRAPHY ROUGHNESS}

To display noise in a STM feedback system in the frequency domain, a spectrum analyzer can be used. Here, we focus on the integrated noise, determined from topography images with the roughness value $R_{a}$. The dependence between roughness and ideal feedback parameters (gain parameters) were studied in Ref. 15, but while imaging surfaces by STM and included tip-sample noise. Therefore, variations of the roughness as a function of the gain parameters are justified.

The roughness value $R_{a}$ is mainly used for quality monitoring ${ }^{16}$ and it summarizes the averaged surface height variations of a profile corresponding to

$$
R_{a}=\frac{1}{N} \sum_{i=1}^{N}\left|z_{i}-\langle z\rangle\right|
$$

with $N$ as the maximal number of pixel in an image and $\left|z_{i}-\langle z\rangle\right|$ as the deviation in the profile from the average value $\langle z\rangle$.

The acquisition of topography images was possible through the implementation of the SPMS, which closed the feedback loop as in a typical STM feedback system. The outgoing $z$ signal from the STM controller was plugged to the $1 \mathrm{M} \Omega$ connection of the SPMS, as shown in Fig. 2. The incoming voltage signal from the $I / V$ converter was connected to the tunnel current input of the STM controller. The recorded topography images were analyzed by the software GWYDDION. ${ }^{17}$

In the following, the dependence between the roughness value $R_{a}$ and the gain parameters of the STM controller was investigated. In our experiments with the SPMS we choose a 


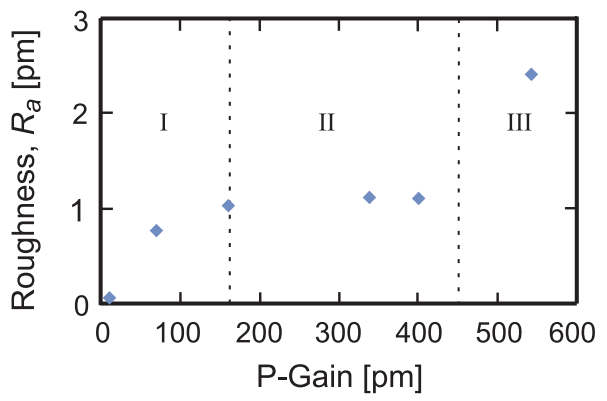

FIG. 3. The roughness $R_{a}$ of the topography as a function of the P-Gain is shown. Low P-Gain values show a strong increase of $R_{a}$ (Region I) as the feedback system cannot fully zero the error signal. In Region II, the PGain values show a saturation of $R_{a}$. For high P-Gain values (Region III), the feedback system starts to oscillate and the apparent roughness $R_{a}$ strongly increases.

tunnel current setpoint of $I_{\text {set }}=0.1 \mathrm{nA}$. The used SPM controller offered two gain parameters, a proportional gain (PGain) and a time constant (T). The time constant was kept constant at $600 \mu \mathrm{s}$, which was a normal value in measurements without the SPMS. In the following the unit of the PGain value is given in $\mathrm{pm}$. The P-Gain was varied between $10 \mathrm{pm}$ and $540 \mathrm{pm}$. The roughness values $R_{a}$ dependent on the P-Gain values are shown in Fig. $3 .{ }^{18}$ With low P-Gain values (Region I), the feedback system is only able to partially control the error signal. The $R_{a}$ values increase strongly with the ability of the controller to control the error signal. For medium P-Gain values (Region II), the feedback system is able to fully control the error signal. The $R_{a}$ value saturates around $1.1 \mathrm{pm}$, but still slightly changes its magnitude caused by a longer transient oscillation time. A high P-Gain value (Region III) results in a drastic increase of the roughness value with $R_{a}=2.4 \mathrm{pm}$. Here, the feedback system is oscillating and strong variations in the topography occur.

A comparison of different STM controllers is only possible if an optimal P-Gain value can be equally determined for different STM controllers. The optimal P-Gain value is located at the smallest P-Gain value in Region II. At this point, the error signal is minimized and the feedback system does not oscillate.

\section{GAIN ADJUSTMENT BY SETTLING TIME MINIMIZATION}

To compare different STM controllers by the topography roughness, it is necessary to use equivalent gain parameters, which are independent of the units or the number of gain parameters.

For determining an optimal gain parameter, the output response of a rectangular perturbation signal was investigated for the settling time $t_{\text {settling }}$. The settling time $t_{\text {settling }}$ is defined as the time required for a signal to reach its set point with a given error $e .{ }^{19}$ This is schematically shown in Fig. 4(a).

The rectangular perturbation signal was connected to the $10 \mathrm{M} \Omega$ input of the SPMS. The amplitude $V_{r}$ of the rectangular perturbation signal was $50 \mathrm{mV}$ at a frequency of $f=36.6 \mathrm{~Hz}$, which equals to a period time of $T_{p}=1 / f$ $=27.3 \mathrm{~ms}$. One plateau of the rectangluar perturbation signal

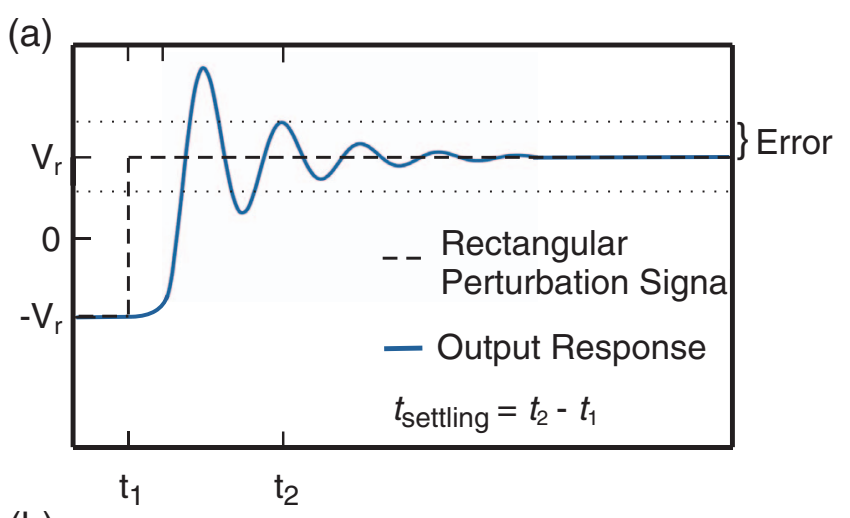

(b)

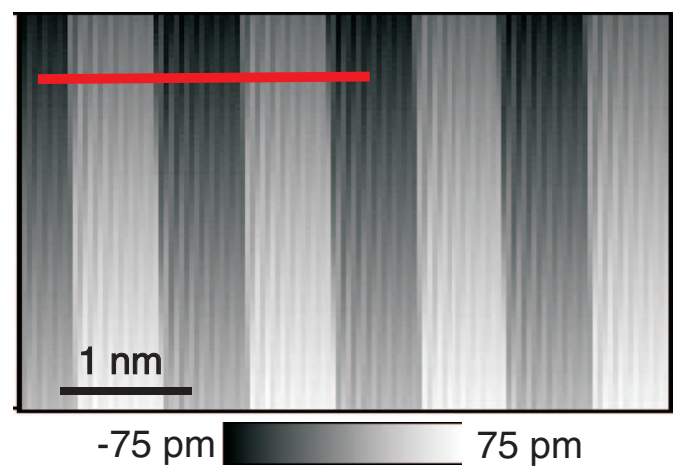

(c)

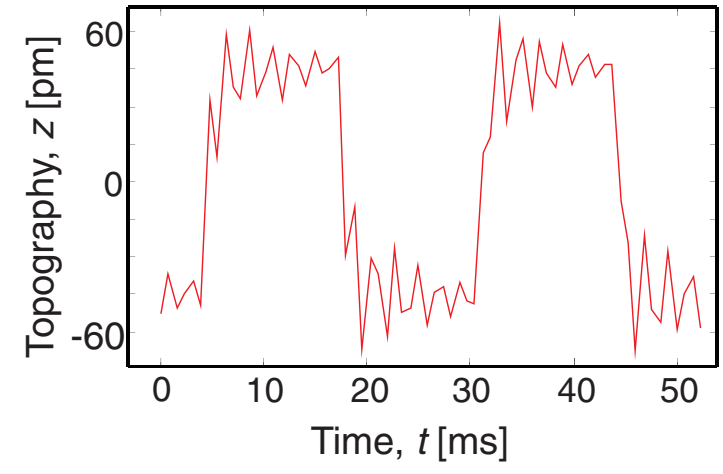

FIG. 4. (a) Schematic of the rectangular perturbation signal and the output response. The settling time $t_{\text {settling }}$ is the time between the rectangular perturbation signal rise and the output response is within a defined error. ${ }^{19}$ (b) Topographic image of the rectangular perturbation signal, with $V_{r}=50 \mathrm{mV}, f=36.6 \mathrm{~Hz}$, and P-Gain $=450 \mathrm{pm}$. (c) Cross section from (b) to extract $t_{\text {settling }}$.

corresponds to $13.7 \mathrm{~ms}$. The frequency was chosen to exclude crosstalk with $50 \mathrm{~Hz}$ noise.

The settling times were extracted from recorded images, Fig. 4(b). A typical cross section is shown in Fig. 4(c). The dimensions of the image divided by the used scan speed $v$ determined the time $t$. For example, a distance of $0.7 \mathrm{~nm}$ (length of a plateau) divided by a scan speed of $50 \mathrm{~nm} / \mathrm{s}$ results in $14 \mathrm{~ms}$.

By minimizing the settling time $t_{\text {settling }}$, we found the optimal gain parameter. The error $e$ was set to approximately $10 \%$. The measurements of $t_{\text {settling }}$ are summarized in Fig. 5. The settling times dependent on the P-Gain values show a parabola. The outer two points of the parabola were estimated as their settling times took longer than the time of $13.7 \mathrm{~ms}$ corresponding to one plateau of the rectangular perturbation 


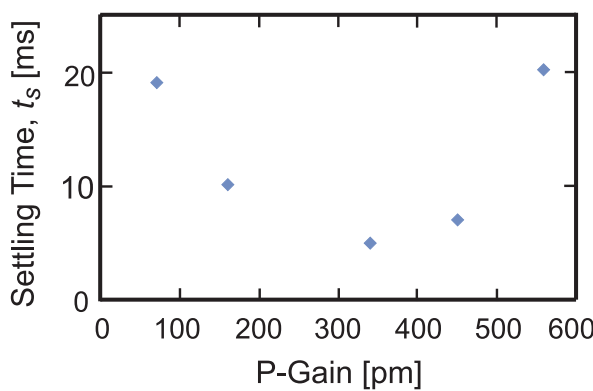

FIG. 5. The optimal P-Gain value was determined by measuring the minimum settling time for a rectangular perturbation signal. The two outer points of the parabola were estimated as there settling times were not within the time according to a plateau of the rectangular perturbation signal. The minimum $\mathrm{P}-\mathrm{Gain}$ is around $340 \mathrm{pm}$.

signal. The minimum of the parabola is around $340 \mathrm{pm}$, which was set for further experiments as the optimal P-Gain value.

\section{COMPARISON OF DIFFERENT STM CONTROLLER}

The optimal gain parameter was determined for two STM controllers, called A and B, by settling time measurements. Controller A was the same as used in the experiments explained in Secs. I-IV. Controller B had only one gain parameter to adjust the settling time. The tunnel current setpoint was kept the same for both controllers. ${ }^{20}$

The topography images for comparison were recorded without a rectangular perturbation signal, but with the adjusted optimal gain parameter. Therefore, the topography images of the controllers looked flat with differences in surface roughness. The roughness value of controller $\mathrm{A}, R_{a, A}$, is $1.12 \mathrm{pm}$. Controller B had with $R_{a, B}=1.99 \mathrm{pm}$ a topog-

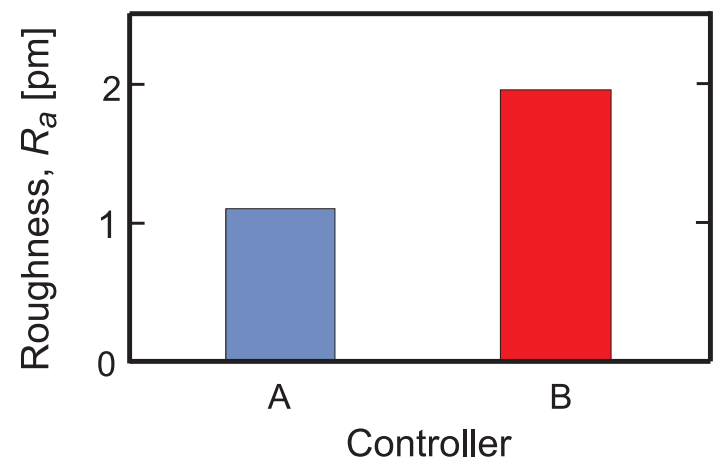

FIG. 6. The roughness values $R_{a}$ are shown for two STM controllers, A and B. The extracted $R_{a}$ for controller A is $1.12 \mathrm{pm}$, which is approximately two times smaller than $R_{a}=1.99 \mathrm{pm}$ for controller B. Controller B was a 20 year old feedback electronic compared to a modern feedback electronic called controller A raphy roughness almost two times higher than controller A. Controller B was a 20 year old STM controller and it was expected that controller A would outperform it (see Fig. 6).

In conclusion, the SPMS enables one to investigate the noise of SPM controllers and allows the comparison of resulting topography roughness of different SPM controllers although the basic feedback parameter (e.g., P-Gain and time constant) are differently specified. This is only possible since no irregularities in the tip-sample junction disturb the measurements.

Furthermore, the SPMS offers the possibility to operate the control electronics under specific and realistic closedfeedback conditions to isolate broken electronic components.

\section{ACKNOWLEDGMENTS}

The authors thank the Deutsche Forschungsgemeinschaft for funding through SFB 689 and the colleagues from the Institute of Experimental and Applied Physics for fruitful discussions. Thanks to A. J. Weymouth and E. Wutscher for proofreading.

${ }^{1}$ G. Binnig, H. Rohrer, C. Gerber, and E. Weibel, Phys. Rev. Lett. 49, 57-61 (1982).

${ }^{2}$ G. Binnig, H. Rohrer, C. Gerber, and E. Weibel, Phys. Rev. Lett. 50, 120 123 (1983).

${ }^{3}$ Y. Kuk and P. J. Silverman, Rev. Sci. Instrum. 60, 165-180 (1989).

${ }^{4}$ Scanning Tunneling Microsocpy, edited by J. A. Stroscio and W. J. Kaiser (Academic Press, 1993).

${ }^{5}$ Noncontact Atomic Force Microscopy, edited by S. Morita, F. J. Giessibl, and R.Wiesendanger (Springer, 2009).

${ }^{6}$ R. García and R. Pérez, Surf. Sci. Rep. 47, 197-301 (2002).

${ }^{7}$ F. J. Giessibl, Rev. Mod. Phys. 75, 943-983 (2003).

${ }^{8}$ I. Ekvall, E. Wahlström, D. Claesson, H. Olin, and E. Olsson, Meas. Sci. Technol. 10, 11 (1999).

${ }^{9}$ G. Eder, S. Schlögl, K. Macknapp, W. M. Heckl, and M. Lackinger, Rev. Sci. Instrum. 82, 033701 (2011).

${ }^{10}$ T. Hofmann, J. Welker, and F. J. Giessibl, J. Vac. Sci. Technol. B 28, C4E28-C4E30 (2010).

${ }^{11}$ T. Wutscher and F. J. Giessibl, Rev. Sci. Instrum. 82, 026106 (2011).

${ }^{12}$ Philips Semiconductors, Datasheet, High-speed diode 1N4148; 1N4448 (2002).

${ }^{13}$ Analog Devices, Datasheet, AD711 (2002).

${ }^{14}$ Fairchild Semiconductor, Datasheet, FDH/FDLL300/A/333 (2000).

${ }^{15}$ M. Aguilar, A. I. Oliva, and E. Anguiano, Europhys. Lett. 46, 442-447 (1999).

${ }^{16}$ E. S. Gadelmawla, M. M. Koura, T. M. A. Maksoud, I. M. Elewa, and H. H. Soliman, J. Mater. Process. Technol. 123, 133-145 (2002).

${ }^{17}$ D. Nečas and P. Klapetek, Cent. Eur. J. Phys. 10, 181 (2012).

${ }^{18} \mathrm{~J}$. Niebauer, "Aufbau und Erprobung eines Kelvinreglers und Messungen auf Graphen mit dem qPlus Sensor," Diploma thesis (University of Regensburg, 2012).

${ }^{19}$ T.-T. Tay, I. Mareels, and J. B. Moore, High Performance Control (Birkhäuser, 1997).

${ }^{20}$ The conversion factor for the used piezo actuator (tube scanner) in $\mathrm{nm} / \mathrm{V}$ should have a fixed value, otherwise the topography between different controllers values cannot be compared in a useful manner. 